\title{
Localized operator partitioning method for electronic excitation energies in the time-dependent density functional formalism
}

\author{
Jayashree Nagesh, ${ }^{1}$ Michael J. Frisch, ${ }^{2}$ Paul Brumer, ${ }^{1}$ and Artur F. Izmaylov ${ }^{1,3}$ \\ 1) Chemical Physics Theory Group, Department of Chemistry, University of Toronto, Toronto, ON M5S 3H6, \\ Canada \\ ${ }^{2)}$ Gaussian, Inc., 340 Quinnipiac Street, Building 40, Wallingford, Connecticut 06492, \\ U.S.A \\ ${ }^{3)}$ Department of Physical and Environmental Sciences, University of Toronto Scarborough, Toronto, ON M1C 1A4, \\ Canada
}

(Dated: 16 October 2018)

We extend the localized operator partitioning method (LOPM) [J. Nagesh, A.F. Izmaylov, and P. Brumer, J. Chem. Phys. 142, 084114 (2015)] to the time-dependent density functional theory (TD-DFT) framework to partition molecular electronic energies of excited states in a rigorous manner. A molecular fragment is defined as a collection of atoms using Stratman-Scuseria-Frisch atomic partitioning. A numerically efficient scheme for evaluating the fragment excitation energy is derived employing a resolution of the identity to preserve standard one- and two-electron integrals in the final expressions. The utility of this partitioning approach is demonstrated by examining several excited states of two bichromophoric compounds: 9-((1-naphthyl $)$ - methyl $)$ - anthracene and 4-((2-naphthyl $)$ - methyl $)$-benzaldehyde. The LOPM is found to provide nontrivial insights into the nature of electronic energy localization that are not accessible using simple density difference analysis.

\section{INTRODUCTION}

Understanding and controlling electronic energy transfer (EET) is at the heart of effective utilization of solar energy, ${ }^{3}$ and and efficient light harvesting in biomolecular processes ${ }^{4}$. Therefore obtaining insights in EET mechanisms through first principle modeling is of paramount importance. A first-principles study of EET is challenging because both electronic and nuclear degrees of freedom are usually involved (See for example Ref. $\underline{5}$ ). Moreover, a quantitative investigation of EET requires a computational tool that allows one to monitor how much electronic energy is located on a molecular fragment.

When molecular fragments are well separated in space, various versions of the Förster theory can be successfully used for the EET investigation. ${ }^{6}-\underline{-}$ In contrast, monitoring electronic energy of a fragment becomes particularly challenging for flexible polymeric systems where electronic wave-function components of chromophoric fragments can overlap and thus interact relatively strongly (e.g., via $\pi-\pi$ stacking). Such overlaps make traditional approaches based on the Förster ${ }^{6}$ and Dexter ${ }^{9}$ theories inadequate because of perturbative treatment of the inter-chromophore interactions and lack of rigorous spatial definition of the donor and acceptor. To avoid these deficiencies one can use techniques where multi-electronic state dynamics is obtained in the diabatic representation with a construction of diabatic states maximizing excitation localization but spanning the whole system. ${ }^{10}$ However, such diabatic techniques do not quantify the amount of electronic energy located on a particular fragment of the molecule. 11

Recently, the localized operator partitioning method (LOPM) has been developed in order to address the problem of electronic energy partitioning independent of the degree of separation or strength of interaction between chromophoric fragments. The LOPM is immune to the described problems since it formulates the partitioned energy electronic Hamiltonian that provides the corresponding local electronic energy as an expectation value $E_{p}(t)=\left\langle\Psi(t)\left|H_{p}\right| \Psi(t)\right\rangle$ using the total system wavefunction $|\Psi(t)\rangle$. That is, the LOPM philosophy is based on the quantum requirement that an operator, here $H_{p}$, corresponds to every measurable. Note that both fully quantum electron-nuclear wave-function or electron only wave-function from mixed quantum-classical approaches can be used in the $E_{p}(t)$ expression. Using any approach to define the spatial volume around a nucleus as an atom 12 and grouping such atoms into fragments 13,14 the LOPM provides non-perturbative energy partitioning with atomic resolution. This technique can be applied for investigating the stationary states as a first step toward a full dynamical description. Any electronic structure technique can be used in the LOPM, but to provide a proof of concept illustration the simplest excited state generation method, configuration interaction singles (CIS) have been used in Ref. 2. It is well-known however that CIS overestimates excitation energies compared to time-dependent density functional theory (TDDFT) methods when compared to experiment. Progress in linear response Kohn-Sham (KS) TD-DFT over last couple of decades has shown that it is feasible to solve for the ground and singly excited valence states of the full system ${ }^{15,16}$ with a good balance between accuracy and computational cost. Thus in this paper we develop the LOPM within the TD-DFT formalism and obtain spatially partitioned ground and excited state electronic energies, leading to first-principles-based approach into EET in realistic molecules.

The remainder of this paper is organized as follows. 
Section III overviews the theory behind the LOPM and describes its extension to the density functional formalism for ground and excited states. In Sec. III we describe details of implementation. Section IV reports the application of the LOPM to two bichromophoric organic molecules where singlet-singlet and triplet-triplet EET processes have been previously studied. Section V concludes by providing a summary and outlook for the LOPM.

\section{THEORY}

\section{A. Localized operator partitioning method}

We briefly review the LOPM from Refs. 2 and 1 to establish the notation for various quantities. Our starting point is the electronic Hamiltonian obtained after the Born-Oppenheimer separation

$$
\begin{aligned}
\hat{H}_{e} & =\sum_{m} h\left(\mathbf{r}_{m}\right)+\sum_{m>n} \frac{1}{\left|\mathbf{r}_{m}-\mathbf{r}_{n}\right|} \\
& +\sum_{k>l} \frac{Z_{k} Z_{l}}{\left|\mathbf{R}_{k}-\mathbf{R}_{l}\right|}
\end{aligned}
$$

where

$$
h\left(\mathbf{r}_{m}\right)=-\frac{1}{2} \nabla_{m}^{2}-\sum_{k} \frac{Z_{k}}{\left|\mathbf{R}_{k}-\mathbf{r}_{m}\right|}
$$

is the one-electron part, $\mathbf{r}_{m}$ and $\mathbf{R}_{k}$ are electronic and nuclear coordinates, $\nabla_{m}^{2}$ is an electronic Laplacian, and $Z_{k}$ 's are nuclear charges. ${ }^{17}$ The nuclear-nuclear repulsion term in Eq. (1) does not contribute to the electronic excitation energies that are of the main interest to EET and therefore will be neglected below.

For stationary states of $\hat{H}_{e}$, the partitioned electronic Hamiltonian $\hat{H}_{e}^{(p)}$ of subsystem $p$ is defined as ${ }^{2}$

$$
\hat{H}_{e}^{(p)}=\sum_{m} \theta_{p}\left(\mathbf{r}_{m}\right) h\left(\mathbf{r}_{m}\right)+\frac{1}{2} \sum_{m \neq n} \frac{\theta_{p}\left(\mathbf{r}_{m}\right)}{\left|\mathbf{r}_{m}-\mathbf{r}_{n}\right|},
$$

where

$$
\theta_{p}\left(\mathbf{r}_{m}\right)= \begin{cases}1 & \text { if } \mathbf{r}_{m} \in p \\ 0 & \text { otherwise }\end{cases}
$$

is the one-electron projection operator for subsystem $p$. For electronic eigenstates $\Psi_{I}$ of $\hat{H}_{e}$, subsystem energies are given by $E_{p}^{(I)}=\left\langle\Psi_{I}\left|\hat{H}_{e}^{(p)}\right| \Psi_{I}\right\rangle$. Similarly, we define average subsystem electron populations for each electronic state $I$ as

$$
\mathcal{N}_{I}^{(p)}=\left\langle\Psi_{I}\left|\sum_{m} \theta_{p}\left(\mathbf{r}_{m}\right)\right| \Psi_{I}\right\rangle .
$$

Owing to the completeness relation for one-electron projection operators 1

$$
\sum_{p} \theta_{p}(\mathbf{r})=\mathbf{1}_{\mathbf{r}}
$$

the subsystem properties $E_{p}^{(I)}$ and $\mathcal{N}_{p}^{(I)}$ are additive and are summed to corresponding total unpartitioned values.

\section{B. Partitioning in Kohn-Sham density functional theory}

The ground state Kohn-Sham density functional theory (KS-DFT) energy is given by

$$
E[\rho]=\left\langle\Phi_{\mathrm{KS}}\left|\hat{H}_{e}\right| \Phi_{\mathrm{KS}}\right\rangle_{S}+E_{\mathrm{xc}}^{(\mathrm{DFT})}[\rho],
$$

where $\left|\Phi_{\mathrm{KS}}\right\rangle$ is the KS determinant, subscript $S$ refers to scaling and introducing range separation in the HartreeFock (HF) exchange part,

$$
\begin{gathered}
\left\langle\Phi_{\mathrm{KS}}\left|\hat{H}_{e}\right| \Phi_{\mathrm{KS}}\right\rangle_{S}=\int d \mathbf{r}\left[h(\mathbf{r}) \rho\left(\mathbf{r}, \mathbf{r}^{\prime}\right)\right]_{\mathbf{r}=\mathbf{r}^{\prime}} \\
+\sum_{n>m} \frac{Z_{n} Z_{m}}{\left|\mathbf{R}_{m}-\mathbf{R}_{n}\right|}+J[\rho]+K[\rho] \\
J[\rho]=\frac{1}{2} \int d \mathbf{r} d \mathbf{r}^{\prime} \frac{\rho\left(\mathbf{r}^{\prime}\right) \rho(\mathbf{r})}{\left|\mathbf{r}^{\prime}-\mathbf{r}\right|} \\
K[\rho]=-\sum_{m}^{3} \frac{\alpha_{m}}{4} \int d \mathbf{r} d \mathbf{r}^{\prime} \frac{\left|\rho\left(\mathbf{r}, \mathbf{r}^{\prime}\right)\right|^{2}}{\left|\mathbf{r}-\mathbf{r}^{\prime}\right|} \mathcal{O}_{m}\left(\mathbf{r}, \mathbf{r}^{\prime}\right)
\end{gathered}
$$

where $\mathcal{O}_{m}\left(\mathbf{r}, \mathbf{r}^{\prime}\right)$ represents long-range $\left[\operatorname{erf}\left(\gamma\left|\mathbf{r}-\mathbf{r}^{\prime}\right|\right)\right]$, short-range $\left[\operatorname{erfc}\left(\gamma\left|\mathbf{r}-\mathbf{r}^{\prime}\right|\right)\right]$ or full-range $\left(\mathbf{1}_{\mathbf{r}, \mathbf{r}^{\prime}}\right)$ operators with appropriate scaling factor $\alpha_{m}, \rho\left(\mathbf{r}, \mathbf{r}^{\prime}\right)$ is the oneparticle density matrix corresponding to $\left|\Phi_{\mathrm{KS}}\right\rangle$ and $\rho\left(\mathbf{r}^{\prime}\right)$ is its diagonal part. The pure DFT exchange-correlation part is

$$
E_{\mathrm{xc}}^{(\mathrm{DFT})}[\rho]=\int d \mathbf{r}\left[e_{x}\left(\rho(\mathbf{r}) ;\left\{\alpha_{m}\right\}, \gamma\right)+e_{c}(\rho(\mathbf{r}))\right],
$$

where $e_{x}$ and $e_{c}$ are the exchange-correlation energy densities, the former parametrically depends on the scaling constants $\alpha_{i}$ and $\gamma$. Partitioning the scaled Hamiltonian in Eq. (8) is done by partitioning the electronic Hamiltonian and then scaling the HF exchange component

$$
\begin{aligned}
\left\langle\Phi_{\mathrm{KS}}\left|\hat{H}_{e}^{(p)}\right| \Phi_{\mathrm{KS}}\right\rangle_{S}= & \int d \mathbf{r} \theta_{p}(\mathbf{r})\left[h(\mathbf{r}) \rho\left(\mathbf{r}, \mathbf{r}^{\prime}\right)\right]_{\mathbf{r}=\mathbf{r}^{\prime}} \\
& +J^{(p)}[\rho]+K^{(p)}[\rho] \\
J^{(p)}[\rho]= & \frac{1}{2} \int d \mathbf{r} d \mathbf{r}^{\prime} \frac{\rho\left(\mathbf{r}^{\prime}\right) \rho(\mathbf{r}) \theta_{p}(\mathbf{r})}{\left|\mathbf{r}^{\prime}-\mathbf{r}\right|} \\
K^{(p)}[\rho]= & -\sum_{m}^{3} \frac{\alpha_{m}}{4} \int d \mathbf{r} d \mathbf{r}^{\prime} \frac{\left|\rho\left(\mathbf{r}, \mathbf{r}^{\prime}\right)\right|^{2}}{\left|\mathbf{r}-\mathbf{r}^{\prime}\right|} \\
& \times \mathcal{O}_{m}\left(\mathbf{r}, \mathbf{r}^{\prime}\right) \theta_{p}(\mathbf{r}) .
\end{aligned}
$$

The pure DFT exchange-correlation part is partitioned term-wise by using the additivity of integration and $\theta_{p}(\mathbf{r})$ completeness [Eq. (6)]

$$
E_{\mathrm{xc}}^{(\mathrm{DFT}, \mathrm{p})}[\rho]=\int d \mathbf{r} \theta_{p}(\mathbf{r})\left[e_{x}\left(\rho(\mathbf{r}) ;\left\{\alpha_{i}\right\}, \gamma\right)+e_{c}(\rho(\mathbf{r}))\right]
$$

Therefore the partitioned KS ground state energy is

$$
E^{(p)}[\rho]=\left\langle\Phi_{\mathrm{KS}}\left|\hat{H}_{e}^{(p)}\right| \Phi_{\mathrm{KS}}\right\rangle_{S}+E_{\mathrm{xc}}^{(\mathrm{DFT}, \mathrm{p})}[\rho] .
$$




\section{Partitioning in time-dependent density functional theory}

Casida's equations provide excitation energies, $\omega_{I}$, in a linear response regime as a solution of the generalized eigenvalue problem ${ }^{18}$

$$
\left(\begin{array}{cc}
\mathbf{A} & \mathbf{B} \\
\mathbf{B}^{*} & \mathbf{A}^{*}
\end{array}\right)\left(\begin{array}{l}
\mathbf{X} \\
\mathbf{Y}
\end{array}\right)_{I}=\omega_{I}\left(\begin{array}{cc}
\mathbf{1} & \mathbf{0} \\
\mathbf{0} & -\mathbf{1}
\end{array}\right)\left(\begin{array}{l}
\mathbf{X} \\
\mathbf{Y}
\end{array}\right)_{I}
$$

where $\mathbf{X}$ and $\mathbf{Y}$ stand for excitation and de-excitation coefficient vectors respectively. The matrix elements of $\mathbf{A}$ and $\mathbf{B}$ written in the KS molecular orbital (MO) basis are

$$
\begin{aligned}
A_{i a, j b} & =\delta_{i j} \delta_{a b}\left(\epsilon_{a}-\epsilon_{i}\right)+\left\langle i j\left|f_{\mathrm{xc}}^{(\mathrm{DFT})}\right| a b\right\rangle \\
& +\langle i j \mid a b\rangle-\sum_{m}\langle i a \mid j b\rangle_{m},
\end{aligned}
$$

and

$$
B_{i a, j b}=\left\langle i j\left|f_{\mathrm{xc}}^{(\mathrm{DFT})}\right| b a\right\rangle+\langle i j \mid b a\rangle-\sum_{m}\langle i j \mid a b\rangle_{m},
$$

where $\epsilon_{a}$ and $\epsilon_{i}$ are KS MO energies, $f_{\mathrm{xc}}^{(\mathrm{DFT})}$ is the exchange-correlation kernel

$$
f_{\mathrm{xc}}^{(\mathrm{DFT})}\left(\mathbf{r}, \mathbf{r}^{\prime}\right)=\frac{\delta^{2} E_{\mathrm{xc}}^{(\mathrm{DFT})}}{\delta \rho(\mathbf{r}) \delta \rho\left(\mathbf{r}^{\prime}\right)},
$$

and

$$
\begin{aligned}
\langle r s \mid t u\rangle & =\int d \mathbf{r} d \mathbf{r}^{\prime} \phi_{r}(\mathbf{r}) \phi_{t}(\mathbf{r}) \frac{1}{\left|\mathbf{r}-\mathbf{r}^{\prime}\right|} \phi_{s}\left(\mathbf{r}^{\prime}\right) \phi_{u}\left(\mathbf{r}^{\prime}\right), \quad(21) \\
\langle r s \mid t u\rangle_{m} & =\alpha_{m} \int d \mathbf{r} d \mathbf{r}^{\prime} \phi_{r}(\mathbf{r}) \phi_{t}(\mathbf{r}) \frac{\mathcal{O}_{m}\left(\mathbf{r}, \mathbf{r}^{\prime}\right)}{\left|\mathbf{r}-\mathbf{r}^{\prime}\right|} \phi_{s}\left(\mathbf{r}^{\prime}\right) \phi_{u}\left(\mathbf{r}^{\prime}\right)
\end{aligned}
$$

are the Coulomb and scaled exchange integrals respectively in Dirac's notation. Here we use labels $i, j, \ldots$ for occupied; $a, b, \ldots$ for unoccupied, and $r, s, \ldots$ to indicate either type of orbitals.

It is convenient to recast the excitation energy as

$$
\omega_{I}=\left(\begin{array}{ll}
\mathbf{X}^{\dagger} & \mathbf{Y}^{\dagger}
\end{array}\right)_{I}\left(\begin{array}{cc}
\mathbf{A} & \mathbf{B} \\
\mathbf{B}^{*} & \mathbf{A}^{*}
\end{array}\right)\left(\begin{array}{l}
\mathbf{X} \\
\mathbf{Y}
\end{array}\right)_{I} .
$$

The origin of this quadratic form is the second variation of energy with respect to the one-electron density

$$
\omega_{I}=E_{I}-E_{0}=\int d \mathbf{r} \int d \mathbf{r}^{\prime} \frac{\delta^{2} E[\rho]}{\delta \rho(\mathbf{r}) \delta \rho\left(\mathbf{r}^{\prime}\right)} \rho_{I}^{(1)}(\mathbf{r}) \rho_{I}^{(1)}\left(\mathbf{r}^{\prime}\right),
$$

where $\rho_{I}^{(1)}(\mathbf{r})$ is the $I$ th first order density response to an external potential variation, e.g., exciting laser field. Since the total energy contains orbital dependent part, $\left\langle\Phi_{\mathrm{KS}}\left|H_{e}\right| \Phi_{\mathrm{KS}}\right\rangle_{S}$, and the exchange correlation part, $E_{\mathrm{xc}}^{(\mathrm{DFT})}[\rho]$, their variations are usually done differently using variations with respect to the KS orbitals for the $\left\langle\Phi_{\mathrm{KS}}\left|H_{e}\right| \Phi_{\mathrm{KS}}\right\rangle_{S}$ part and variation with respect to the density for the $E_{\mathrm{xc}}^{(\mathrm{DFT})}[\rho]$ part

$$
\begin{aligned}
\omega_{I} & =\sum_{i j a b}\left\{U_{i a} V_{j b}\right. \\
& \left.\times \int d \mathbf{r} \int d \mathbf{r}^{\prime} \frac{\delta^{2}\left\langle\Phi_{\mathrm{KS}}\left|H_{e}\right| \Phi_{\mathrm{KS}}\right\rangle_{S}}{\delta \phi_{i}(\mathbf{r}) \delta \phi_{j}\left(\mathbf{r}^{\prime}\right)} \phi_{a}(\mathbf{r}) \phi_{b}\left(\mathbf{r}^{\prime}\right)\right\} \\
& +\int d \mathbf{r} \int d \mathbf{r}^{\prime} \frac{\delta^{2} E_{\mathrm{xc}}^{(\mathrm{DFT})}[\rho]}{\delta \rho(\mathbf{r}) \delta \rho\left(\mathbf{r}^{\prime}\right)} \rho_{I}^{(1)}(\mathbf{r}) \rho_{I}^{(1)}\left(\mathbf{r}^{\prime}\right),
\end{aligned}
$$

where $\rho_{I}^{(1)}(\mathbf{r})=\sum_{i a} X_{i a} \phi_{i}^{*}(\mathbf{r}) \phi_{a}(\mathbf{r})+Y_{i a} \phi_{a}^{*}(\mathbf{r}) \phi_{i}(\mathbf{r})$, and $U_{i a} V_{j b}$ are four possible products $X_{i a}^{*} X_{j b}, Y_{i a}^{*} X_{j b}$, $X_{i a}^{*} Y_{j b}$, and $Y_{i a}^{*} Y_{j b}$ depending on which part of $\delta^{2}\left\langle\Phi_{\mathrm{KS}}\left|H_{e}\right| \Phi_{\mathrm{KS}}\right\rangle_{S} / \delta \phi_{i}(\mathbf{r}) \delta \phi_{j}\left(\mathbf{r}^{\prime}\right)$, bra and/or ket, the orbital variation is taking place. Equation (25) maps solving Casida's equation to finding normal modes in density variations with the second variation of energy with respect to the density as the electronic energy Hessian.

Our partitioning approach can be straightforwardly generalized to Eq. (25) by switching the order between the partitioning operation and the second variation

$$
\begin{aligned}
\omega_{I}^{(p)} & =\int d \mathbf{r} d \mathbf{r}^{\prime} \mathcal{P}\left\{\frac{\delta^{2} E}{\delta \rho(\mathbf{r}) \delta \rho\left(\mathbf{r}^{\prime}\right)}\right\} \rho_{I}^{(1)}(\mathbf{r}) \rho_{I}^{(1)}\left(\mathbf{r}^{\prime}\right) \\
& =\int d \mathbf{r} d \mathbf{r}^{\prime}\left\{\frac{\delta^{2} \mathcal{P}\{E\}}{\delta \rho(\mathbf{r}) \delta \rho\left(\mathbf{r}^{\prime}\right)}\right\} \rho_{I}^{(1)}(\mathbf{r}) \rho_{I}^{(1)}\left(\mathbf{r}^{\prime}\right)
\end{aligned}
$$

$\mathcal{P}\{E\}$ 's variation is done in the same way as for the total energy expression. This leads to the following expression in terms of $\mathrm{KS}$ orbitals

$$
\omega_{I}^{(p)}=\left(\begin{array}{ll}
\mathbf{X}^{\dagger} & \mathbf{Y}^{\dagger}
\end{array}\right)_{I}\left(\begin{array}{cc}
\mathbf{A}^{(p)} & \mathbf{B}^{(p)} \\
\mathbf{B}^{(p)^{*}} & \mathbf{A}^{(p)^{*}}
\end{array}\right)\left(\begin{array}{c}
\mathbf{X} \\
\mathbf{Y}
\end{array}\right)_{I},
$$

where

$$
\begin{aligned}
A_{i a, j b}^{(p)} & =\delta_{i j} F_{a b}^{(p)}-\delta_{a b} F_{i j}^{(p)} \\
& +\left\langle i j\left|f_{\mathrm{xc}}^{(\mathrm{DFT})}\right| a b\right\rangle^{(p)}+\langle i j \mid a b\rangle^{(p)}-\sum_{m}\langle i a \mid j b\rangle_{m}^{(p)} \\
B_{i a, j b}^{(p)} & =\left\langle i j\left|f_{\mathrm{xc}}^{(\mathrm{DFT})}\right| b a\right\rangle^{(p)}+\langle i j \mid b a\rangle^{(p)}-\sum_{m}\langle i j \mid a b\rangle_{m}^{(p)} .
\end{aligned}
$$

Here, $F_{r s}^{(p)}$ stands for the partitioned KS Fock matrix,

$$
\begin{aligned}
F_{s t}^{(p)} & =h_{s t}^{(p)}+\sum_{i}\left\{2\langle i s \mid i t\rangle^{(p)}-\sum_{m}\langle i i \mid s t\rangle_{m}^{(p)}\right\} \\
h_{s t}^{(p)} & =\int d \mathbf{r} \theta_{p}(\mathbf{r}) \phi_{s}^{*}(\mathbf{r}) h(\mathbf{r}) \phi_{t}(\mathbf{r})
\end{aligned}
$$


and

$$
\begin{aligned}
\left\langle r s\left|f_{\mathrm{xc}}^{(\mathrm{DFT})}\right| t u\right\rangle^{(p)} & =\int d \mathbf{r} d \mathbf{r}^{\prime} \theta_{p}(\mathbf{r}) \frac{\delta^{2} E_{\mathrm{xc}}^{(\mathrm{DFT})}}{\delta \rho(\mathbf{r}) \delta \rho\left(\mathbf{r}^{\prime}\right)} \\
& \times \phi_{r}(\mathbf{r})^{*} \phi_{t}(\mathbf{r}) \phi_{s}\left(\mathbf{r}^{\prime}\right)^{*} \phi_{u}\left(\mathbf{r}^{\prime}\right), \\
\langle r s \mid t u\rangle_{m}^{(p)} & =\alpha_{m} \int d \mathbf{r} d \mathbf{r}^{\prime} \theta_{p}(\mathbf{r}) \frac{\mathcal{O}_{m}\left(\mathbf{r}, \mathbf{r}^{\prime}\right)}{\left|\mathbf{r}-\mathbf{r}^{\prime}\right|} \\
& \times \phi_{r}(\mathbf{r})^{*} \phi_{t}(\mathbf{r}) \phi_{s}\left(\mathbf{r}^{\prime}\right)^{*} \phi_{u}\left(\mathbf{r}^{\prime}\right), \\
\langle r s \mid t u\rangle^{(p)} & =\int d \mathbf{r} d \mathbf{r}^{\prime} \theta_{p}(\mathbf{r}) \frac{1}{\left|\mathbf{r}-\mathbf{r}^{\prime}\right|} \\
& \times \phi_{r}(\mathbf{r})^{*} \phi_{t}(\mathbf{r}) \phi_{s}\left(\mathbf{r}^{\prime}\right)^{*} \phi_{u}\left(\mathbf{r}^{\prime}\right) .
\end{aligned}
$$

We note that although the full Fock matrix is diagonal, its partitioned counterpart has non-zero off-diagonal elements.

\section{IMPLEMENTATION}

\section{A. Resolution of identity}

For efficient implementation of the partitioned energies within the TD-DFT formalism we use the localized atomic orbital (AO) Gaussian basis set $\left(\phi_{\mu}, \phi_{\nu} \ldots\right)$ to evaluate the fragment ground and excited state energies. This allows us to employ numerous screening techniques in generating $\mathrm{AO}$ one- and two-electron integrals that are contracted on-the-fly with corresponding densities. 16 The direct scheme leads to AO counterparts of integrals in Eqs. (32)-(35). Partitioning the exchange-correlation part in $f_{\mathrm{xc}}^{(\mathrm{DFT})}$ integrals [Eq. (33)] is done by eliminating the quadrature points located outside of the subsystem region. Straightforward partitioning of the nuclearelectron attraction and electron-electron repulsion integrals would involve modification of the Boys integrals to accommodate the altered shape of the partitioned AOs and thus would create computational difficulties. To circumvent this problem we replace the partitioning operator $\hat{\theta}_{p}$ by its projected form employing the resolution of identity $(\mathrm{RI})$ technique ${ }^{2}$ with the projection operator

$$
\hat{\mathbb{1}}=\sum_{\mu \nu}|\mu\rangle\left(S^{-1}\right)_{\nu \mu}\langle\nu|,
$$

where $\left(S^{-1}\right)_{\nu \mu}$ are matrix elements of the inverse of the AO overlap matrix $S_{\mu \nu}=\langle\mu \mid \nu\rangle$ and $|\mu\rangle,|\nu\rangle$ are AO basis functions. Then the projected form of $\hat{\theta}_{p}$ is

$$
\begin{aligned}
& \tilde{\theta}_{p}=\hat{\Perp} \hat{\theta}_{p} \hat{\Perp} \\
& =\sum_{\mu \nu}|\mu\rangle L_{\mu \nu}^{(p)}\langle\nu|,
\end{aligned}
$$

where

$$
\begin{aligned}
L_{\mu \nu}^{(p)} & =\sum_{\mu_{1} \nu_{1}}\left(S^{-1}\right)_{\mu \mu_{1}} S_{\mu_{1} \nu_{1}}^{(p)}\left(S^{-1}\right)_{\nu_{1} \nu} \\
S_{\mu_{1} \nu_{1}}^{(p)} & =\left\langle\mu_{1}\left|\hat{\theta}_{p}\right| \nu_{1}\right\rangle \\
& =\int d \mathbf{r} \phi_{\mu_{1}}(\mathbf{r}) \theta_{p}(\mathbf{r}) \phi_{\nu_{1}}(\mathbf{r}) .
\end{aligned}
$$

Thus, to obtain the projected partitioning $\tilde{\theta}_{p}$, the partitioned overlap matrix $S_{\mu \nu}^{(p)}$ elements are evaluated as weighted sums

$$
S_{\mu \nu}^{(p)}=\sum_{k \in \Omega_{p}} \sum_{i} w\left(\mathbf{r}_{i}\right) p_{k}\left(\mathbf{r}_{i}\right) \phi_{\mu}\left(\mathbf{r}_{i}\right) \phi_{\nu}\left(\mathbf{r}_{i}\right)
$$

where $\Omega_{p}$ denotes a group of atoms representing fragment $p$ in the molecule, with $p_{k}\left(\mathbf{r}_{i}\right)$ being $k^{\text {th }}$ atom's spatial partition function in the Stratman-Scuseria-Frisch atomic partitioning scheme, and $w\left(\mathbf{r}_{i}\right)$ is the normalized quadrature weight associated with grid point $\mathbf{r}_{i}$ in a spherical quadrature scheme $\underline{19}$

Using the projected partitioning we recast the partitioned one-electron operator $\hat{h}$ AO matrix elements as a matrix product of standard one-electron integrals and overlap matrices

$$
\left\langle\mu\left|\hat{h} \tilde{\theta}_{p}\right| \nu\right\rangle=\sum_{\lambda \sigma}\langle\mu|\hat{h}| \lambda\rangle S_{\lambda \sigma}^{-1} S_{\sigma \nu}^{(p)}
$$

similar products are obtained for the two-electron $\mathrm{AO}$ integrals

$$
\begin{aligned}
& \langle\mu \lambda \mid \nu \sigma\rangle^{(p)}=\sum_{\mu_{1} \nu_{1}} S_{\mu \mu_{1}}^{(p)}\left(S^{-1}\right)_{\mu_{1} \nu_{1}}\left\langle\nu_{1} \lambda \mid \nu \sigma\right\rangle \\
& \langle\mu \lambda \mid \nu \sigma\rangle_{m}^{(p)}=\sum_{\mu_{1} \nu_{1}} S_{\mu \mu_{1}}^{(p)}\left(S^{-1}\right)_{\mu_{1} \nu_{1}}\left\langle\nu_{1} \lambda \mid \nu \sigma\right\rangle_{m} .
\end{aligned}
$$

Although there is a difference in results of partitioning by $\tilde{\theta}_{p}$ and $\hat{\theta}_{p}$, this is not an issue because we consider $\tilde{\theta}_{p}$ as our primary partitioning operator. This projected partitioning is more convenient in implementation and gives exactly the same partitioned density matrices as a non-projected version.

Thus, the projecting partitioning operator $\tilde{\theta}_{p}$ [Eq. (37)] allows us to use standard integrals in both one- and twoelectron contributions [Eqs. (43)-(45)], and thus to bypass the problem of partitioning the Boys integrals.

\section{B. Working equations for TD-DFT partitioned excitation energies in the $A O$ representation}

Before introducing partitioning in working energy expressions we provide those for the unpartitioned ground and excited states in the $\mathrm{AO}$ representation. The ground 
state KS-DFT energy for the closed shell case is given by

$$
\begin{aligned}
E_{K S}^{(0)} & =\sum_{\mu \nu} P_{\mu \nu}^{(0)} h_{\mu \nu}+E_{\mathrm{xc}}^{(\mathrm{DFT})} \\
& +\sum_{\mu \nu \lambda \sigma}\left\{2 P_{\mu \nu}^{(0)} P_{\lambda \sigma}^{(0)}\langle\mu \lambda \mid \nu \sigma\rangle-P_{\mu \sigma}^{(0)} P_{\lambda \nu}^{(0)} \sum_{m}\langle\mu \lambda \mid \nu \sigma\rangle_{m}\right\}
\end{aligned}
$$

where $P_{\mu \nu}^{(0)}=\sum_{i} C_{\mu i} C_{\nu i}$ is the $\mathrm{AO}$ density matrix and $\left\{C_{\mu p}\right\}$ are the KS MO coefficients.

The excited state energies $\omega_{I}$ are evaluated using the symmetrized and anti-symmetrized AO transition densities $\mathbf{T}^{ \pm}$

$$
T_{\mu \nu}^{ \pm(I)}=\sum_{i a}\left(X_{i a}^{(I)} \pm Y_{i a}^{(I)}\right)\left(C_{\mu i} C_{\nu a} \pm C_{\mu a} C_{\nu a}\right) .
$$

Assuming a closed shell singlet state, we transform Eq. (25) into the AO basis and group the one- and twoelectron terms to obtain

$$
\begin{aligned}
\omega_{I} & =E_{o r b}^{(I)}+E_{x c}^{(I)}+E_{2 e}^{(I)}, \\
E_{o r b}^{(I)} & =\sum_{\mu \nu} R_{\mu \nu}^{(I)} h_{\mu \nu} \\
& +\sum_{\mu \nu \lambda \sigma}\left\{J_{\mu \nu \lambda \sigma}^{(I)}\langle\mu \lambda \mid \nu \sigma\rangle+\sum_{m} K_{\mu \nu \lambda \sigma}^{(I)}\langle\mu \lambda \mid \nu \sigma\rangle_{m}\right\} \\
E_{x c}^{(I)} & =\sum_{\mu \nu \lambda \sigma} D_{\mu \nu \lambda \sigma}^{(I)}\left\langle\mu \lambda\left|f_{\mathrm{xc}}^{(\mathrm{DFT})}\right| \nu \sigma\right\rangle \\
E_{2 e}^{(I)} & =\sum_{\mu \nu \lambda \sigma}\left\{D_{\mu \nu \lambda \sigma}^{(I)}\langle\mu \lambda \mid \nu \sigma\rangle+\frac{1}{2} \Gamma_{\mu \nu \lambda \sigma}^{(I)} \sum_{m}\langle\mu \lambda \mid \nu \sigma\rangle_{m}\right\}
\end{aligned}
$$

and

$$
\begin{aligned}
R_{\mu \nu}^{(I)} & =\sum_{i j a b} U_{i a}^{(I)} U_{j b}^{(I)}\left\{\delta_{i j} C_{\mu b} C_{\nu a}-\delta_{a b} C_{\mu i} C_{\nu j}\right\}, \\
J_{\mu \nu \lambda \sigma}^{(I)} & =2 P_{\mu \nu}^{(0)} R_{\lambda \sigma}^{(I)}+2 R_{\mu \nu}^{(I)} P_{\lambda \sigma}^{(0)}, \\
K_{\mu \nu \lambda \sigma}^{(I)} & =-P_{\mu \sigma}^{(0)} R_{\lambda \nu}^{(I)}-R_{\mu \sigma}^{(I)} P_{\lambda \nu}^{(0)}, \\
D_{\mu \nu \lambda \sigma}^{(I)} & =T_{\mu \nu}^{+(I)} T_{\sigma \lambda}^{+(I)}+T_{\lambda \sigma}^{+(I)} T_{\mu \nu}^{+(I)}, \\
\Gamma_{\mu \nu \lambda \sigma}^{(I)} & =\left\{T_{\mu \nu}^{+(I)} T_{\sigma \lambda}^{+(I)}+T_{\mu \lambda}^{+(I)} T_{\nu \sigma}^{+(I)}+T_{\lambda \sigma}^{+(I)} T_{\nu \mu}^{+(I)}\right. \\
& +T_{\lambda \mu}^{+(I)} T_{\sigma \nu}^{+(I)}+T_{\mu \lambda}^{-(I)} T_{\nu \sigma}^{-(I)}-T_{\mu \sigma}^{-(I)} T_{\lambda \nu}^{-(I)} \\
& \left.+T_{\lambda \mu}^{-(I)} T_{\sigma \nu}^{-(I)}-T_{\lambda \nu}^{-(I)} T_{\mu \sigma}^{-(I)}\right\} .
\end{aligned}
$$

Therefore all components of excitation energy [Eq. (48)] can be efficiently generated by contracting 2-index density-like quantities with the standard one- and twoelectron integrals, 16,20

The ground state energy is partitioned as

$$
\begin{aligned}
& E_{K S}^{(0, p)}=\sum_{\mu \nu} \tilde{P}_{\mu \nu}^{(0, p)} h_{\mu \nu}+E_{\mathrm{xc}}^{(\mathrm{DFT}, \mathrm{p})} \\
& +\sum_{\mu \nu \lambda \sigma}\left\{2 \tilde{P}_{\mu \nu}^{(0, p)} P_{\lambda \sigma}^{(0)}\langle\mu \lambda \mid \nu \sigma\rangle-\tilde{P}_{\mu \sigma}^{(0, p)} P_{\lambda \nu}^{(0)} \sum_{m}\langle\mu \lambda \mid \nu \sigma\rangle_{m}\right\}
\end{aligned}
$$

where the first and last two terms are obtained by contracting the matrix elements from Eqs. (43), (44) and (45) with $\mathbf{P}^{(0)}$ as

$$
\begin{aligned}
\sum_{\mu \nu} P_{\mu \nu}^{(0)}\left\langle\mu\left|\tilde{\theta}_{p} \hat{h}\right| \nu\right\rangle & =\sum_{\mu \nu \lambda \sigma} P_{\mu \nu}^{(0)} S_{\mu \lambda}^{(p)}\left(S^{-1}\right)_{\lambda \sigma} h_{\sigma \nu} \\
& =\sum_{\nu \sigma} \tilde{P}_{\nu \sigma}^{(0, p)} h_{\sigma \nu} \\
\sum_{\mu \nu \lambda \sigma} 2 P_{\mu \nu}^{(0)} P_{\lambda \sigma}^{(0)}\langle\mu \lambda \mid \nu \sigma\rangle^{(p)} & =\sum_{\mu \nu \lambda \sigma} 2 P_{\mu \nu}^{(0)} P_{\lambda \sigma}^{(0)} \\
& \times \sum_{\mu_{1} \nu_{1}} S_{\mu \mu_{1}}^{(p)}\left(S^{-1}\right)_{\mu_{1} \nu_{1}}\left\langle\nu_{1} \lambda \mid \nu \sigma\right\rangle \\
& =\sum_{\nu_{1} \lambda \nu \sigma} 2 \tilde{P}_{\nu \nu_{1}}^{(0, p)} \tilde{P}_{\lambda \sigma}^{(0)}\left\langle\nu_{1} \lambda \mid \nu \sigma\right\rangle .
\end{aligned}
$$

Following a similar procedure for the excitation energies, we obtain

$$
\begin{aligned}
\omega_{I}^{(p)} & =E_{o r b}^{(I, p)}+E_{x c}^{(I, p)}+E_{2 e}^{(I, p)}, \\
E_{o r b}^{(I, p)} & =\sum_{\mu \nu} \tilde{R}_{\mu \nu}^{(I, p)} h_{\mu \nu} \\
& +\sum_{\mu \nu \lambda \sigma}\left\{\tilde{J}_{\mu \nu \lambda \sigma}^{(I, p)}\langle\mu \lambda \mid \nu \sigma\rangle+\tilde{K}_{\mu \nu \lambda \sigma}^{(I, p)} \sum_{m}\langle\mu \lambda \mid \nu \sigma\rangle_{m}\right\} \\
E_{x c}^{(I, p)} & =\sum_{\mu \nu \lambda \sigma} \tilde{D}_{\mu \nu \lambda \sigma}^{(I, p)}\left\langle\mu \lambda\left|f_{\mathrm{xc}}^{(\mathrm{DFT})}\right| \nu \sigma\right\rangle, \\
E_{2 e}^{(I, p)} & =\sum_{\mu \nu \lambda \sigma}\left\{\tilde{D}_{\mu \nu \lambda \sigma}^{(I, p)}\langle\mu \lambda \mid \nu \sigma\rangle+\frac{1}{2} \tilde{\Gamma}_{\mu \nu \lambda \sigma}^{(I, p)} \sum_{m}\langle\mu \lambda \mid \nu \sigma\rangle_{m}\right\},
\end{aligned}
$$

where

$$
\begin{aligned}
\tilde{R}_{\mu \nu}^{(I, p)} & =\sum_{\lambda \sigma} R_{\mu \lambda}^{(I)} S_{\lambda \sigma}^{(p)}\left(S^{-1}\right)_{\sigma \nu} \\
\tilde{J}_{\mu \nu \lambda \sigma}^{(I, p)} & =2 \tilde{P}_{\mu \nu}^{(0, p)} R_{\lambda \sigma}^{(I)}+2 \tilde{R}_{\mu \nu}^{(I, p)} P_{\lambda \sigma}^{(0)} \\
\tilde{K}_{\mu \nu \lambda \sigma}^{(I, p)} & =-\tilde{P}_{\mu \sigma}^{(0, p)} R_{\lambda \nu}^{(I)}-\tilde{R}_{\mu \sigma}^{(I, p)} P_{\lambda \nu}^{(0)}
\end{aligned}
$$

and using symmetrized $\tilde{\mathbf{T}}^{+(I, p)}$ and anti-symmetrized $\tilde{\mathbf{T}}^{-(I, p)}$ partitioned transitioned densities

$$
\begin{aligned}
\tilde{T}_{\mu \nu}^{(I, p)} & =\sum_{\lambda \sigma} T_{\mu \lambda}^{(I)} S_{\lambda \sigma}^{(p)}\left(S^{-1}\right)_{\sigma \nu}, \\
\tilde{T}_{\mu \nu}^{ \pm(I, p)} & =\frac{1}{2}\left[\tilde{T}_{\mu \nu}^{(I, p)} \pm \tilde{T}_{\nu \mu}^{(I, p)}\right],
\end{aligned}
$$

the two-electron densities in $E_{H x c}^{(I, p)}$ and $E_{2 e}^{(I, p)}$ are

$$
\begin{aligned}
\tilde{D}_{\mu \nu \lambda \sigma}^{(I, p)} & =\tilde{T}_{\mu \nu}^{+(I, p)} T_{\sigma \lambda}^{+(I)}+\tilde{T}_{\lambda \sigma}^{+(I, p)} T_{\mu \nu}^{+(I)}, \\
\tilde{\Gamma}_{\mu \nu \lambda \sigma}^{(I, p)} & =\left\{\tilde{T}_{\mu \nu}^{+(I, p)} T_{\sigma \lambda}^{+(I)}+\tilde{T}_{\mu \lambda}^{+(I, p)} T_{\nu \sigma}^{+(I)}+\tilde{T}_{\lambda \sigma}^{+(I, p)} T_{\nu \mu}^{+(I)}\right. \\
& +\tilde{T}_{\lambda \mu}^{+(I, p)} T_{\sigma \nu}^{+(I)}+\tilde{T}_{\mu \lambda}^{-(I, p)} T_{\nu \sigma}^{-(I)}-\tilde{T}_{\mu \sigma}^{-(I, p)} T_{\lambda \nu}^{-(I)} \\
& \left.+\tilde{T}_{\lambda \mu}^{-(I, p)} T_{\sigma \nu}^{-(I)}-\tilde{T}_{\lambda \nu}^{-(I, p)} T_{\mu \sigma}^{-(I)}\right\} .
\end{aligned}
$$


FIG. 1. A1N and Closs-M molecules with fragment definitions used in this work. Atoms in blue correspond to the donor in an EET process.

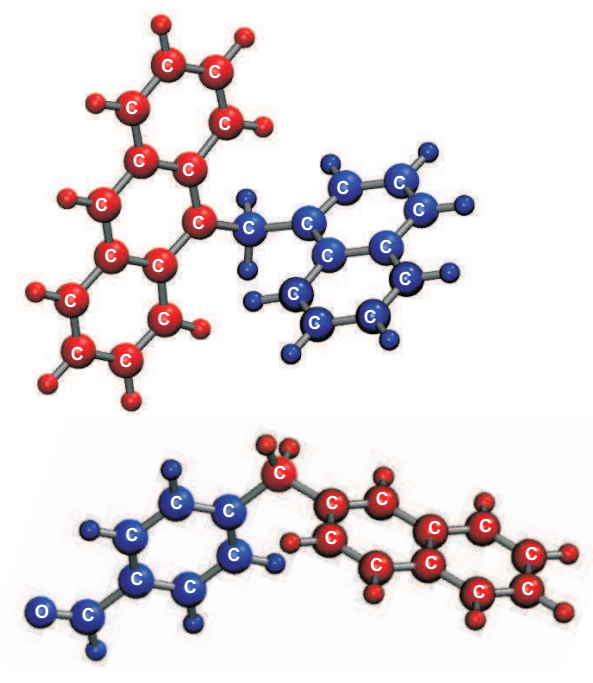

Thus, using the projected local electronic partitioning allows us to formulate all computationally intense partitioned quantities as a product of standard Gaussian integrals contracted with various densities. These partitioned energy equations have been implemented in the Gaussian suite of programs. 21

\section{RESULTS AND DISCUSSION}

We apply the resulting formulae to partition energies of two bichromophores: $9-((1-$ naphthyl $)-$ methyl $)$-anthracene $(\mathrm{A} 1 \mathrm{~N})^{22}$ and 4-((2-naphthyl $)$ - methyl $)$ - benzaldehyde (Closs-M $)^{23}$ (Fig. 1), whose singlet-singlet and triplet-triplet EET have been investigated experimentally. All TD-DFT calculations used 6-31G(d) basis set for energy calculations and the RI expansion in the partioning operator definition. The unrelaxed one-electron densities for excited states were used to analyze various properties of these states. A spherical quadrature scheme consisting of a pruned grid of 75 radial shells and 302 angular points per shell per atom ${ }^{24}$ was employed to evaluate numerical partitioned overlap integrals and DFT contributions.

Total excitation energies of A1N: Table 1 presents full system excited state energies using TD-HF, pure, hybrid, and long-range corrected (LRC) functionals for $\mathrm{A} 1 \mathrm{~N}$. The obtained results can be compared with available gas-phase experimental estimates obtained from fluorescence excitation spectra. ${ }^{22}$ The original spectra are vibrationally resolved and to obtain estimates corresponding to vertical electronic transitions we used intensityweighted sums $\omega_{J}^{(\exp )}=\sum_{n} I_{n} \Delta E_{n},{ }^{29}$ where $\Delta E_{n}$ are the vibronic peak positions for the ground to $J$ th excited electronic state transition, and $I_{n}$ are corresponding nor-
FIG. 2. Orbital energy diagram illustrating occurrence of negative energy differences between partitioned virtual $\epsilon_{a}$ and occupied $\epsilon_{i}$ energies: (left) occupied $\phi_{i}$ and virtual $\phi_{a}$ orbitals are localized on donor and acceptor fragments respectively; (right) occupied $\phi_{i}$ orbital is localized on the B-fragment and virtual $\phi_{a}$ orbital is delocalized.

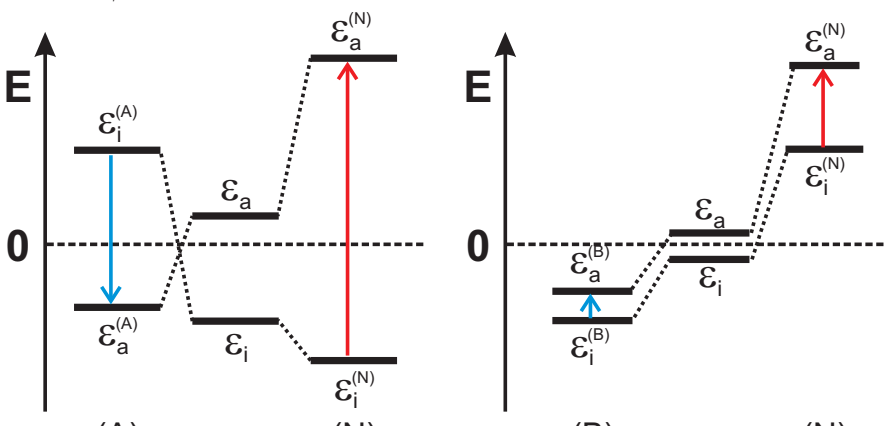

(A)

$(\mathrm{N})$

(B)

$(\mathrm{N})$

malized intensities. Based on vertical excitation energies of bright transitions, PBE1PBE has the best agreement with experimental estimates. Nature of excited states was further analyzed using the atomic partitioning to obtain a fraction of electronic charge transferred from the anthracene upon the excitation, $\Delta \mathcal{N}_{I}^{(A)}$ [Eq. (5)] in Table II. This analysis shown that the dark states between two bright states in pure and hybrid functionals have a charge transfer (CT) character. This is consistent with previous studies revealing a problem of energy underestimation for $\mathrm{CT}$ states due to inaccuracies in a treatment of electron-hole attraction in CT states. This problem is somewhat reduced in the long-range corrected functionals, CAM-B3LYP and $\omega$ B97X-D, $, 30,31$ but their bright state excitations deviate from experiment by an amount more than that of PBE1PBE.

Partitioned excitation energies of A1N: LOPM characterization of $\mathrm{A} 1 \mathrm{~N}$ excited state energies in the form of ratios between partitioned and total energy differences, $\omega_{I}^{(p)} / \omega_{I}$, is presented in Table II. The magnitude of these ratios reflects the extent of excitation energy localization on the fragment. Table II] also provides more common analysis of excited states based on the difference between unrelaxed one-electron excited densities and the ground state density, we will refer to this approach as the difference density (DD) analysis. Combined with an evaluation of the charge difference on a fragment for the ground and excited states ${ }^{32}$ DD is usually used to characterize excitation localization. 31,33 For localized excitations DD analysis usually predicts changes in density on the fragment where partitioned energy is localized according to the LOPM. However, there could be exceptions to this simple observation as we will see below.

As discussed in detail in Ref. 2, the LOPM approach can produce negative ratios due to de-excitations on a fragment $\omega_{I}^{(p)}<0$. Even from a variational point of view, the result $\omega_{I}^{(p)}<0$ is not surprising because partitioned 
TABLE I. Comparison of TD-DFT and TD-HF excitation energies, $\omega_{I}(\mathrm{eV})$, and oscillator strengths $\left(f_{I}\right)$ in A1N using different functionals for 5 lowest excited states; the experimental vertical transition estimates are $3.34 \mathrm{eV}\left(\mathrm{S}_{0}-\mathrm{S}_{1}\right)$ and $4.01 \mathrm{eV}\left(\mathrm{S}_{0}-\mathrm{S}_{2}\right)$. The results for each functional are at the corresponding optimized geometry for the ground state.

\begin{tabular}{ccccccccccc}
\hline \hline State & \multicolumn{2}{c}{ LSDA } & \multicolumn{2}{c}{ PBE1PBE 26} & \multicolumn{2}{c}{ CAM-B3LYP 27} & \multicolumn{2}{c}{$\omega \mathrm{B} 97 \mathrm{X}-\mathrm{D}{ }^{28}$} & \multicolumn{2}{c}{ HF } \\
\hline & $\omega_{I}$ & $f_{I}$ & $\omega_{I}$ & $f_{I}$ & $\omega_{I}$ & $f_{I}$ & $\omega_{I}$ & $f_{I}$ & $\omega_{I}$ & $f_{I}$ \\
\hline 1 & 2.65 & 0.0039 & 3.26 & 0.1060 & 3.58 & 0.1411 & 3.59 & 0.1402 & 4.01 & 0.1988 \\
2 & 2.85 & 0.0636 & 3.62 & 0.0007 & 4.10 & 0.0009 & 4.10 & 0.0011 & 4.63 & 0.0008 \\
3 & 2.96 & 0.0024 & 3.88 & 0.0002 & 4.62 & 0.0065 & 4.66 & 0.0002 & 5.02 & 0.1039 \\
4 & 3.50 & 0.0004 & 3.98 & 0.0011 & 4.70 & 0.0242 & 4.71 & 0.1217 & 5.29 & 0.0004 \\
5 & 3.64 & 0.0006 & 4.45 & 0.1057 & 4.74 & 0.0929 & 4.81 & 0.0026 & 5.79 & 0.0044 \\
\hline
\end{tabular}

TABLE II. Local TD-DFT and TD-HF populations and excitation energies in A1N: $\Delta \mathcal{N}_{I}^{(A)}=\mathcal{N}_{I}^{(A)}-\mathcal{N}_{0}^{(A)} ; \sum_{p} \mathcal{N}_{I}^{(p)}=N_{e}$ for all I; In A1N, $N_{e}=168$ and $\mathcal{N}_{0}^{(A)}=92.93$. DD refers to visual identification from difference density analysis of each state's unrelaxed excited state density at density isosurface value of $4 \times 10^{-4}$ a.u.

\begin{tabular}{|c|c|c|c|c|c|}
\hline Functional & $I$ & $\Delta \mathcal{N}_{I}^{(A)}$ & $\overline{\omega_{I}^{(A)} / \omega_{I}}$ & $\overline{\omega_{I}^{(N)} / \omega_{I}}$ & DD \\
\hline \multirow{5}{*}{ LSDA } & 1 & 0.91 & -76.66 & 77.66 & CT: $(\mathrm{N}) \rightarrow(\mathrm{A})$ \\
\hline & 2 & -0.01 & 2.27 & -1.27 & Local on (A) \\
\hline & 3 & -0.90 & 69.18 & -68.18 & $\mathrm{CT}:(\mathrm{A}) \rightarrow(\mathrm{N})$ \\
\hline & 4 & 0.94 & -59.16 & 60.16 & $\mathrm{CT}:(\mathrm{N}) \rightarrow(\mathrm{A})$ \\
\hline & 5 & -0.03 & 2.77 & -1.77 & Local on (A) \\
\hline \multirow{5}{*}{ PBE1PBE } & 1 & 0.01 & 1.07 & -0.07 & Local on (A) \\
\hline & 2 & 0.91 & -56.06 & 57.06 & $\mathrm{CT}:(\mathrm{N}) \rightarrow(\mathrm{A})$ \\
\hline & 3 & -0.71 & 41.90 & -40.90 & $\mathrm{CT}:(\mathrm{A}) \rightarrow(\mathrm{N})$ \\
\hline & 4 & -0.22 & 13.22 & -12.22 & Delocalized \\
\hline & 5 & 0.01 & -0.54 & 1.54 & Local on $(\mathrm{N})$ \\
\hline \multirow{5}{*}{ CAM-B3LYP } & 1 & 0.00 & 1.28 & -0.28 & Local on (A) \\
\hline & 2 & -0.01 & 1.49 & -0.49 & Local on (A) \\
\hline & 3 & 0.71 & -34.75 & 35.75 & CT: $(\mathrm{N}) \rightarrow(\mathrm{A})$ \\
\hline & 4 & 0.09 & -4.10 & 5.10 & Delocalized $^{a}$ \\
\hline & 5 & 0.05 & -2.19 & 3.19 & Delocalized $^{a}$ \\
\hline \multirow{5}{*}{$\omega \mathrm{B} 97 \mathrm{X}-\mathrm{D}$} & 1 & 0.00 & 1.32 & -0.32 & Local on (A) \\
\hline & 2 & -0.01 & 1.51 & -0.51 & Local on (A) \\
\hline & 3 & 0.09 & -4.65 & 5.65 & Delocalized $^{a}$ \\
\hline & 4 & 0.01 & -0.59 & 1.59 & Local on $(\mathrm{N})$ \\
\hline & 5 & 0.72 & -34.54 & 35.54 & $\mathrm{CT}:(\mathrm{N}) \rightarrow(\mathrm{A})$ \\
\hline \multirow{5}{*}{$\mathrm{HF}$} & 1 & 0.00 & 1.43 & -0.43 & Local on (A) \\
\hline & 2 & -0.01 & 1.51 & -0.51 & Local on (A) \\
\hline & 3 & 0.00 & 0.01 & 0.99 & Local on (N) \\
\hline & 4 & 0.00 & -0.15 & 1.15 & Local on (N) \\
\hline & 5 & 0.00 & 1.10 & -0.10 & Local on (A) \\
\hline
\end{tabular}

a The majority of the difference density is on the N-fragment.

energies $E_{I}^{(p)}$ are expectation values of the partitioned Hamiltonian and thus do not have to be ordered in the same way as the corresponding energies $E_{I}$ obtained as per the variational principle applied to the full system. The largest de-excitations are observed in charge-transfer (CT) states; to understand their origin it suffices to consider their main components from the orbital energy difference part $\Delta E_{I} \approx \epsilon_{a}-\epsilon_{i}$, where orbitals $\phi_{a}$ and $\phi_{i}$ are localized on different chromophores. Following the example given in Fig. 2(left) where $\phi_{i}$ is localized on the naphthalene $(\mathrm{N})$ fragment it is easy to predict that the one-electron part of $\epsilon_{i}$ upon partitioning will be almost
TABLE III. Local TD-DFT populations and excitation energies in Closs-M using the $\omega$ B97X-D functional: $\Delta \mathcal{N}_{I}^{(B)}=$ $\mathcal{N}_{I}^{(B)}-\mathcal{N}_{0} ; \sum_{p} \mathcal{N}_{I}^{(p)}=N_{e}$ for all I; In Closs-M, $N_{e}=130$ and $\mathcal{N}_{0}^{(B)}=55.00$. DD refers to the visual result from difference density analysis of each state's unrelaxed excited state density at density isosurface value of $4 \times 10^{-4}$ a.u.

\begin{tabular}{cccrrrl}
\hline \hline$I$ & $\omega_{I}(e V)$ & Spin & $\Delta \mathcal{N}_{I}^{(B)}$ & $\omega_{I}^{(B)} / \omega_{I}$ & $\omega_{I}^{(N)} / \omega_{I}$ & DD \\
\hline 1 & 2.80 & $\mathrm{~T}$ & 0.00 & 0.13 & 0.87 & Local on (N) \\
2 & 3.30 & $\mathrm{~T}$ & 0.00 & 1.92 & -0.92 & Local on (B) \\
3 & 3.37 & $\mathrm{~T}$ & -0.01 & -1.18 & 2.18 & Local on (B) \\
4 & 3.93 & $\mathrm{~S}$ & -0.01 & -1.02 & 2.02 & Local on (B) \\
5 & 4.69 & $\mathrm{~T}$ & 0.00 & 0.01 & 0.99 & Local on (N) \\
\hline
\end{tabular}

completely belong to the N-fragment because of the orbital density localization. On the other hand, the N-part of the Coulomb contribution for this orbital energy

$$
\begin{aligned}
J^{(N)}\left(\rho_{i}, \rho_{0}\right) & =\int d \mathbf{r} d \mathbf{r}^{\prime} \frac{\rho_{i}\left(\mathbf{r}^{\prime}\right) \rho_{0}(\mathbf{r})\left(\theta_{N}\left(\mathbf{r}^{\prime}\right)+\theta_{N}(\mathbf{r})\right)}{\left|\mathbf{r}^{\prime}-\mathbf{r}\right|} \\
& =\frac{1}{2}\left[J\left(\rho_{i}^{(N)}, \rho_{0}\right)+J\left(\rho_{i}, \rho_{0}^{(N)}\right)\right]
\end{aligned}
$$

will be significantly reduced due to the averaging of a large $J\left(\rho_{i}^{(N)}, \rho_{0}\right)$ and small $J\left(\rho_{i}, \rho_{0}^{(N)}\right)$ components. Here, $\rho_{i}$ and $\rho_{i}^{(N)}$ are unpartitioned and partitioned orbital densities, and $\rho_{0}$ and $\rho_{0}^{(N)}$ are corresponding total ground state densities. The reduction of $J\left(\rho_{i}, \rho_{0}^{(N)}\right)$ compared to $J\left(\rho_{i}^{(N)}, \rho_{0}\right)$ and $J\left(\rho_{i}, \rho_{0}\right)$ takes place because the total density is always delocalized and its partitioned counterpart is significantly smaller. This reduction of the Coulomb component in $\epsilon_{i}^{(N)}$ leads to a decrease of $\epsilon_{i}^{(N)}$ compare to $\epsilon_{i}$. Due to the additivity of LOPM scheme, $\epsilon_{i}^{(A)}$ experiences an increase. The opposite trend is observed for $\epsilon_{a}$ due to a different localization of orbital $\phi_{a}$. Overall this produces a large excitation and de-excitation on the N- and A-fragments respectively [see Fig. 2(left)].

Interestingly, for CT excitations, the fragment losing electronic charge becomes excited. This is related to a destabilization of the electron donating fragment since the electron departs from the occupied orbital that is below the Fermi level.

Excited states in Closs-M: To avoid spurious lowenergy CT states we have used the $\omega$ B97X-D func- 
tional for this system. Table III shows the partitioned excitation energies of low-lying triplet and singlet states in the Closs-M molecule. All excitation energies are well-localized, but results of the DD analysis disagree with those of the LOPM for states 3 and 4. These states have similar distributions of single-electron excitation/de-excitation coefficients and only differ by the spin multiplicity. An examination of the dominant coefficients reveals that the occupied MOs are localized on the benzaldehyde (B) fragment while the virtual MOs are delocalized over the whole molecule. This leads to larger partitioned Coulomb repulsion in $\epsilon_{a}^{(N)}$ than in $\epsilon_{i}^{(N)}$ that can be seen from Eq. (173) and its counterpart for $\rho_{a}$ : $J\left(\rho_{i}, \rho_{0}^{(N)}\right)<J\left(\rho_{a}, \rho_{0}^{(N)}\right)$ since $\rho_{a}$ is partially localized on the N-fragment, hence it interacts stronger with $\rho_{0}^{(N)}$ than $\rho_{i}$ does; and $J\left(\rho_{i}^{(N)}, \rho_{N}\right)<J\left(\rho_{a}^{(N)}, \rho_{0}\right)$ because $\rho_{i}^{(N)}$ is tiny owing to $\rho_{i}$ localization on the B-fragment. Therefore this greater partitioned Coulomb repulsion makes $\epsilon_{a}^{(N)}-\epsilon_{i}^{(N)}$ positive [see Fig. 2(right)] and determines excitation on the naphthalene.

\section{CONCLUDING REMARKS}

We have developed and implemented a direct scheme of the LOPM to partition the electronic energy of a molecule within the linear response TD-DFT framework using a combination of numerical and analytical integrals involving a RI technique in the KS formalism. We apply the LOPM to the singlet and triplet excited state energies of bichromophore molecules, and find that LOPM is a powerful method that not only partitions electronic energies in an fragment-additive manner, but also provides insights in various one- and two-electron contributions of the fragment energies. It was found that a regular density difference approach to excitation analysis can be qualitatively misleading for the energy partitioning and the LOPM provides a quantitative and reliable alternative.

Further, computed partitioning results do sharply emphasize the well-known need for improved functionals, particularly those that effectively treat charge-transfer excitations, since there is little consistency amongst partitioning results as the functionals are changed. However, since the LOPM methodology developed here is independent of the functionals adopted, the partitioning results are expected to improve as DFT accuracy improves.

\section{ACKNOWLEDGEMENTS}

We acknowledge Mr. Yaser Khan and Professor Moshe Shapiro for stimulating discussions at the beginning of this project. The authors also thank V.N. Staroverov and I.G. Ryabinkin for helpful discussions. J.N. is very grateful for the hospitality of Gaussian Inc. A.F.I. greatly appreciates financial support by the Alfred P. Sloan Foundation and the Natural Sciences and Engineering Re- search Council of Canada (NSERC) through the Discovery Grants Program. P.B. acknowledges financial support from the Air Force Office of Scientific Research under Contract No. FA955-13-1-0005.

${ }^{1}$ Y. Khan and P. Brumer, J. Chem. Phys. 137, 194112 (2012).

${ }^{2}$ J. Nagesh, A. F. Izmaylov, and P. Brumer, J. Chem. Phys. 142, 084114 (2015).

${ }^{3}$ V. May and O. Kühn, Charge and Energy Transfer Dynamics in Molecular Systems (Wiley-VCH, Weinheim, Germany, 2011); 10.1039/c6ee01010e

${ }^{4}$ L. A. Pachon and P. Brumer, Phys. Chem. Chem. Phys. 14, 10094 (2012).

${ }^{5}$ B. R. Landry and J. E. Subotnik, J. Chem. Theory Comput. 10, $4253(2014) \quad$ X. Yang and E. R. Bittner, J. Phys. Chem. A 118, 5196 (2014); S. Banerjee, A. Baiardi, J. Bloino, and V. Barone, J. Chem. Theory and Comp. 12, 2357 (2016).

${ }^{6}$ T. Förster, Rad. Res. Suppl. 2, 326 (1959).

${ }^{7}$ B. P. Krueger, G. D. Scholes, and G. R. Fleming, The Journal of Physical Chemistry B 102, 5378 (1998).

${ }^{8}$ B. Mennucci and C. Curutchet, Phys. Chem. Chem. Phys. 13, 11538 (2011).

${ }^{9}$ D. L. Dexter, J. Chem. Phys. 21, 836 (1953).

${ }^{10}$ R. J. Cave and M. D. Newton, Chem. Phys. Lett. 249, 15 (1996); J. Chem. Phys. 106, 9213 (1997); C. P. Hsu and Z. Q. You, J. Phys. Chem. C 112, 1204 (2008); A. A. Voityuk and N. Rosch, J. Chem. Phys. 117, 5607 (2002); H. C. Chen, Z. Q. You, and C. P. Hsu, 129, 084708 (2008); C. P. Hsu, Acc. Chem. Res. 42, 509 (2009); J. E. Subotnik, J. Vura-Weis, A. J. Sodt, and M. A. Ratner, J. Phys. Chem. A 114, 8665 (2010).

${ }^{11}$ A. F. Izmaylov, J. Nagesh, and P. Brumer, in preparation.

${ }^{12}$ A. M. Pendas, M. A. Blanco, and E. Francisco, J. Comput. Chem. 28, 161 (2006).

${ }^{13}$ Y. Imamura, A. Takahashi, and H. Nakai, J. Chem. Phys. 126, 034103 (2007).

${ }^{14}$ D. R. Alcoba, A. Torre, L. Lain, and R. C. Bochicchio, J. Chem. Phys. 122, 074102 (2005).

${ }^{15}$ R. E. Stratman, G. E. Scuseria, and M. J. Frisch, J. Chem. Phys. 109, 8218 (1998).

${ }^{16}$ G. E. Scuseria, J. Phys. Chem. A 103, 4782 (1999).

${ }^{17}$ Atomic units are used throughout in this work.

${ }^{18}$ M. E. Casida, Recent Advances in Density Functional Methods, Vol. 1 (World Scientific, Singapore, 1995) p. 155.

${ }^{19}$ E. R. Stratman, G. E. Scuseria, and M. J. Frisch, Chem. Phys. Lett. 257, 213 (1996).

${ }^{20}$ A. F. Izmaylov, G. E. Scuseria, and M. J. Frisch, J. Chem. Phys. 125, 104103 (2006).

${ }^{21}$ M. J. Frisch, G. W. Trucks, et al., "Gaussian Development Version, Revision I06," Gaussian Inc. Wallingford CT 2016.

${ }^{22}$ X. Wang, D. H. Levy, M. B. Rubin, and S. Speiser, J. Phys. Chem. A 104, 6558 (2000).

${ }^{23}$ G. L. Closs, P. Piotrowiak, J. M. MacInnis, and G. R. Fleming, J. Am. Chem. Soc. 110, 2652 (1988).

${ }^{24}$ V. I. Lebedev, Zh. Vychisl. Mat. Mat. Fiz. 16, 293 (1976).

${ }^{25}$ S. H. Vosko, L. Wilk, and M. Nusair, "Can. J. Phys." 58, 1200 (1980); P. Hohenberg and W. Kohn, Phys. Rev. 136, B864 (1964); W. Kohn and L. J. Sham, 140, A1133 (1965); J. C. Slater, "The Self-Consistent Field for Molecules and Solids", Vol. 4 (McGraw-Hill, New York, 1974).

${ }^{26}$ C. Adamo and V. Barone, J. Chem. Phys. 110, 6158 (1999).

${ }^{27}$ T. Yanai, D. Tew, and N. Handy, Chem. Phys. Lett. 393, 51 (2004).

${ }^{28}$ J.-D. Chai and M. Head-Gordon, Phys. Chem. Chem. Phys. 10, 6615 (2008).

${ }^{29}$ E. R. Davidson and A. A. Jarzecki, Chem. Phys. Lett. 285, 155 (1998).

${ }^{30}$ A. W. Lange, M. A. Rohrdanz, and J. M. Herbert, J. Phys. Chem. B 112, 6304 (2008). 
${ }^{31}$ D. Jacquemin, E. A. Perpete, G. E. Scuseria, I. Ciofini, and C. Adamo, J. Chem. Theory Comput. 4, 123 (2008); H. Nitta and I. Kawata, Chem. Phys. 405, 93 (2012).

${ }^{32}$ Although in this work we used LOPM calculations for charge differences, we do not expect qualitative changes if other forms of the density partitioning would be used, for example, Mulliken or Löwdin.

${ }^{33}$ K. B. Wiberg, C. M. Hadad, J. B. Foresman, and W. A. Chupka, J. Phys. Chem. 96, 10756 (1992); M. Head-Gordon, A. M. Grana, D. Maurice, and C. A. White, 99, 14261 (1995). 